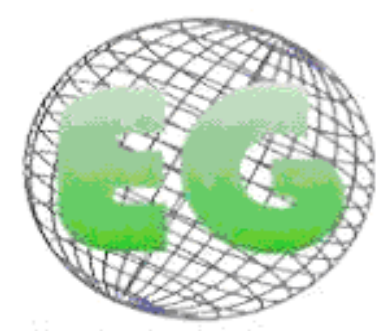

ISSN 1695.6141 $N^{\circ} 26$

\title{
Análisis de la suspensión de cirugía en un hospital docente
}

Análise da suspensão de cirurgias em um hospital de ensino

\begin{abstract}
*Barbosa, MH., "*Miranda Goulart, DM., **Vieira de Andrade, E.,, ${ }^{* *}$ De Mattia, AL.
*Doctora en Enfermería. Profesora Adjunta de la Universidad Federal do Triângulo Mineiro (UFTM). Email: mhelena331@hotmail.com ${ }^{* *}$ Enfermera. ***Doctora en Enfermería. Profesora Adjunta del Departamento de Enfermería Básica de la Escuela de Enfermería de la Universidad Federal de Minas Gerais (UFMG).Brasil.
\end{abstract}

Palabras clave: centros quirúrgicos; enfermería preoperatoria; gerencia
Palavras-chave: centros de cirurgia; enfermagem perioperatória; gerência.

Keywords: surgicenters; perioperative nursing; management.

\section{RESUMEN}

En busca de los índices de mejor calidad, la suspensión de las cirugías han sido examinadas por los servicios y recibido la atención de los investigadores en el campo de la salud. Las altas tasas de cancelación revelan un manejo inadecuado que tienen efectos negativos en el equipo de salud y en la vida del paciente y su familia.

Este estúdio retrospectivo y descriptivo tuvo como objetivo identificar el tipo de suspensión quirúrgica y las razones de este procedimiento en un hospital público de enseñanza. Los datos fueron recolectados de los archivos del Archivo de la Unidad Quirúrgica y Servicio de Archivo Médico y Estadístico del referido hospital.

Hubo una tasa de anulación quirúrgica de $14.14 \%$ y para 1402 (63,78\%) cirugías canceladas no había constancia de la justificación de la suspensión. Las razones para la suspensión de las cirugías fueron: 194 $(8,82 \%)$ por la no hospitalización de los pacientes, $182(8,30 \%)$ por las condiciones clínicas inadecuadas, 179 $(8,14 \%)$ debido a problemas administrativos, $94(4,27 \%)$ por asuntos de asistencia para el cuidados de la salud, $61(2,77 \%)$ por problemas estructurales, $40(1,82 \%)$ por ausencia del pacientes y $46(2.10 \%)$ por otras razones.

Estos resultados señalan a la necesidad de adoptar medidas para garantizar la seguridad del paciente y la calidad del servicio ofrecido.

\section{RESUMO}

Em busca de melhores índices de qualidade, as suspensões de cirurgias têm sido analisadas pelos serviços e recebido atenção por parte de pesquisadores da área de saúde. Altas taxas de cancelamento revelam uma inadequação de gerenciamento que repercutem negativamente na própria equipe de saúde e na vida do paciente e sua família. 
O presente estudo, retrospectivo e descritivo, teve como objetivo identificar a taxa de suspensão cirúrgica e as justificativas para este procedimento em um hospital público de ensino. Os dados foram coletados junto ao Arquivo do Centro Cirúrgico e Serviço de Arquivo Médico e Estatístico do referido hospital.

Verificou-se uma taxa de suspensão cirúrgica de $14,14 \%$ e $1402(63,78 \%)$ cirurgias suspensas não houve registro da justificativa da suspensão. Das suspensões justificadas, $194(8,82 \%)$ foram por não internação do paciente; $182(8,30 \%)$ por condições clínicas do paciente inadequadas; 179 (8,14\%) por problemas administrativos; 94 (4,27\%) por questões assistenciais; 61 (2,77\%) por questões estruturais; $40(1,82 \%)$ por ausência do paciente e $46(2,10 \%)$ por outros motivos.

Estes achados apontam para a necessidade da adoção de medidas que garantam segurança ao paciente e qualidade do serviço oferecido

\section{ABSTRACT}

In the search for better quality indices, the suspensions of surgeries have been examined by and received attention from researchers in the field of health. High cancellation rates reveal an inadequate management that impacts negatively on the health team iself and the patient's life and and that of his or her family.

This retrospective descriptive study aim to identify the rate of surgical suspension and the reasons for this procedure in a public teaching hospital.

Data were collected from the Surgical Center Archive and Medical Archive Service Statistics. There was a rate of $14.14 \%$ surgical cancellations and in $1,402(63.78 \%)$ occasions no records were found that justify the suspension of the surgeries. The results showed the following justifications for the suspensions of the surgery: $194(8.82 \%)$ were non hospitalized patients; $182(8.30 \%)$ for inadequate clinical conditions;, $179(8.14 \%)$ due to administrative problems;, $94(4.27 \%)$ for welfare issues;, 61 (2.77\%) for structural issues; $40(1.82 \%)$ for lack of patients and $46(2.10 \%)$ for other reasons.

These findings point to the need to adopt measures that will ensure patient safety and quality of service offered.

\section{INTRODUCCIÓN}

La mejora continua de la calidad de la ateción a los clientes ha penetrado en los procesos de trabajo en los servicios de salud. La medición del desempeño asistencial está hecha por indicadores que sirven como medida cuantitativa para la reevaluación, replanteamiento y reorganización de las actividades de un servicio, a través de la oferta de ayudas para la toma de decisiones en la gestión de asistencia en la búsqueda de resultados con calidad ${ }^{(1)}$.

En el momento en que se discute la reorganización de los servicios de salud, el Ministerio de Salud define calidad de los servicios de salud como el alto nivel de excelencia profesional, el uso eficiente de recursos, mínimo riesgo para el cliente, alto grado de satisfacción del cliente e impacto final en la salud ${ }^{(2)}$.

El movimiento quirúrgico es considerado una variable que interfiere en los indicadores de calidad y productividad de las instituciones hospitalarias. Se constituye en parámetros de evaluación de la productividad en la sala de cirugía, o sea, tasa de ocupación, tiempo de permanencia, recuperación anestésica, intervalo de tiempo entre cirugías, tasa de retraso y suspensión de cirugía ${ }^{(3)}$.

El tratamiento quirúrgico está indicado en muchas especialidades, provocando el aumento en el número de procedimiento realizados dentro de las instituciones hospitalarias. El Ministerio de Salud señala un aumento de 3,9\% de cirugías electivas en los últimos años ${ }^{(4)}$. En esta perspectiva, la tasa de cancelación de cirugías ha sido el objetivo de estudios y adoptada como un indicador de calidad de la asistencia ${ }^{(5-6,3)}$. 
La suspensión de procedimientos anestésico-quirúrgicos electivos ha sido objeto de investigación no sólo en Brasil, sino también en otros países como Australia, Irlanda, México, Estados Unidos y Reino Unido. Semejantes a los nacionales, esos estudios destacan la alta frecuencia de cancelaciones originadas por cuestiones organizacionales de las instituciones de salud, con consecuente aumento de los costes operacionales y financieros de la Unidad de Centro Quirúrgico, y reducción de la eficiencia y calidad del servicio ofrecido ${ }^{(7)}$.

Se estima que cerca de $60 \%$ de las cancelaciones quirúrgicas electivas son potencialmente evitables, usando técnicas de mejora de la calidad ${ }^{(7)}$.

La utilización de evaluación pre-operatoria, así como la Sistematización de la Asistencia de Enfermería perioperatoria (SAEP) son instrumentos que contribuyen a esta mejora ${ }^{(8)}$.

Estudios nacionales e internacionales $(9,7)$ han mostrado la frecuencia y los motivos de las suspensiones quirúrgicas con el fin de reducir el índice de cancelación y asegurar la calidad de la asistencia para la recuperación de la salud de los pacientes y sus familiares, así como permitir una mejor utilización de los recursos de la institución y su asistencia.

La suspensión de los procedimientos anestésico-quirúrgicos es uno de los cuatro componentes del coste de la calidad, clasificados en costes de fallos internos y externos, costes de la prevención y análisis. Para obtener excelencia, la institución hospitalaria debe estar comprometida continuamente con la resolución, calidad y bajos costes de los procedimientos médicos a través de la eliminación de residuos, habilidad para mejorar el proceso hospitalario (diagnóstico, tratamiento, ingresos, apoyo gerencial), con el aumento de la información y disminución de la permanencia del paciente en el hospital ${ }^{(7,10)}$.

De este modo, la planificación se torna una etapa obligatoria para el funcionamiento operacional dentro de las instituciones de salud para que puedan alcanzar sus objetivos y mantener su responsabilidad para con los pacientes, la familia y la comunidad, una vez que la cancelación de cirugías implique tanto la pérdida y en el mal aprovechamiento de los recursos financieros y de los recursos humanos, como la falta de atención de las necesidades de salud de los clientes asistidos por la institución ${ }^{(5)}$.

Otro aspecto se refiere a las cuestiones relacionadas directamente con las alteraciones psico-emocionales del paciente. Al prepararse para el procedimiento anestésico-quirúrgico, el paciente tiene su foco de atención en todas sus preocupaciones y sus expectativas volcadas para la realización del procedimiento y no para su suspensión, esperando solución para sus problemas tanto por parte del equipo profesional como de la institución que lo atiende $^{(6)}$.

La ansiedad es un estado transitorio, caracterizado por sensaciones desagradables de tensión y aprensión y revela diferencias individuales de reaccionar a situaciones percibidas como amenazadoras, con elevación de la intensidad en el estado de ansiedad ${ }^{(11)}$. En este contexto, la realización de una cirugía depende del trabajo de todo el equipo quirúrgico, siendo el enfermero el elemento que puede presuponer la emoción que el paciente debe sentir, presentando un temor real, el cual se extiende hasta los miembros de la familia. Como elemento clave dentro de la estructura hospitalaria, el enfermero está en condiciones de contribuir a que las cirugías programadas sean realizadas en la fecha marcada y dentro de todas las condiciones de seguridad requeridas ${ }^{(12)}$.

Entre las causas más citadas de suspensión quirúrgica, están las cuestiones administrativas y estructurales ${ }^{(10)}$ y condiciones clínicas inadecuadas ${ }^{(7,12)}$. 
Una constante evaluación de calidad, de la productividad y del impacto de los programas y servicios en la salud de la población es esencial para alertar cuando ocurre desvío de una situación considerada normal o esperada señalando que el proceso en cuestión necesita ser revisado, a fin de impedir la instalación de problemas. De esta manera, el número quirúrgico es un indicador de calidad de la asistencia y de la productividad de la institución ${ }^{(3)}$.

En este contexto, el presente estudio tuvo como objetivo identificar la tasa de suspensión quirúrgica y sus justificativas en un hospital público docente.

\section{MÉTODO}

Se trata de un estudio retrospectivo, con abordaje cuantitativo, realizado en el Centro Quirúrgico de un hospital público, de enseñanza, de gran tamaño, terciario, del municipio de Uberada, Minas Gerais, Brasil. En esta unidad, las especialidades quirúrgicas atendidas incluyen: cabeza y cuello (CCP), cardiaca, digestiva (CAD), general, ortopédica, plástica, proctología, torácica, urología, vascular, ginecológica y obstetricia (GO), neurología, otorrinolaringología, oftalmología y pediátrica.

Se analizaron todos los registros de cirugías canceladas en el periodo de enero de 2006 a diciembre de 2007. La relación de las cirugías programadas y suspendidas con sus respectivas justificaciones fue obtenida en el Servicio de Archivo Médico y Estadístico y el Archivo del Centro Quirúrgico del referido hospital, campo de estudio.

Los datos fueron recogidos después de la aprobación del proyecto de investigación por el Comité de Ética en Investigación de la Universidad Federal del Triangulo Mineiro - UFTM (Parecer $n$ 1014). Se utilizó un instrumento específico para este fin, constituido por dos partes. La primera parte se refería a la identificación del procedimiento propuesto, justificación de la cancelación quirúrgica, periodo (mes y año) y especialidad quirúrgica y la segunda parte, se refería a los datos sociodemográficos (género y edad).

Las justificativas de suspensión del procedimiento anestésico quirúrgico fueron agrupadas utilizando la clasificación propuesta por ${ }^{(7)}$, adaptada, identificándose ochos grupos en este estudio, siendo:

- Grupo 1 - Motivos Asistenciales: inadecuación de la preparación preoperatoria (ausencia de ayunas, administración de medicación contra-indicada en el preoperatorio, ausencia de reserva de sangre y hemoderivados, no realización de evaluación pre-anestésica y análisis complementarios incompletos).

- Grupo 2 - Motivos Administrativos: falta de archivos, ausencia de funcionarios y médicos, superposición de horarios, procedimiento de urgencia en otro paciente en el mismo horario, deficiencia de materiales o equipamientos, cirugías realizadas anteriormente y pacientes sin indicaciones quirúrgicas.

- Grupo 3 - Motivos Estructurales: falta de cama, falta de sala operatoria, servicios de mantenimiento en el Centro Quirúrgico.

- Grupo 4 - Ausencia del paciente.

- Grupo 5 - Condiciones clínicas insatisfactorias en el preoperatorio y/o alteración de conducta médica. 
- Grupo 6 - Injustificadas: sin descripción de los motivos.

- Grupo 7 - No ingresos del paciente debido a no autorización de la guía o del laudo de ingreso, falta de la guía o de tarjeta del Sistema Único de Salud (SUS) o por cuota del SUS sobrepasada.

- Grupo 8 - Otros: se incluyeron en este grupo, retraso del inicio de la cirugía y/o ingreso, error en la programación de la agenda del paciente.

Para el cálculo de la tasa de suspensión del procedimiento anestésico quirúrgico fue utilizada la recomendación por el Ministerio de Salud de Brasil (5), el cual define la tasa de suspensión quirúrgica como el número de cirugías suspendidas, dividido por el total de cirugías programadas, en determinado periodo y multiplicado por 100.

Los datos fueron insertados en una planilla electrónica - Programa $E X C E L^{\circledR}$ for Windows $X P^{\circledast}$ y analizados según estadística descriptiva, en frecuencias absolutas, relativa, media. Los resultados son presentados en forma de graficos.

\section{RESULTADOS}

En el periodo de enero de 2006 a diciembre de 2007 fueron programados 15547 procedimientos anestésico-quirúrgicos y suspendidos 2198, evidenciando una tasa de suspensión quirúrgica de 14,14\%. En el año de 2006 fueron 6935 cirugías programadas y $804(11,60 \%)$ suspendidas y en 2007, fueron 8612 cirugías programadas y $1394(16,19 \%)$ suspendidas (Gráfico 1)

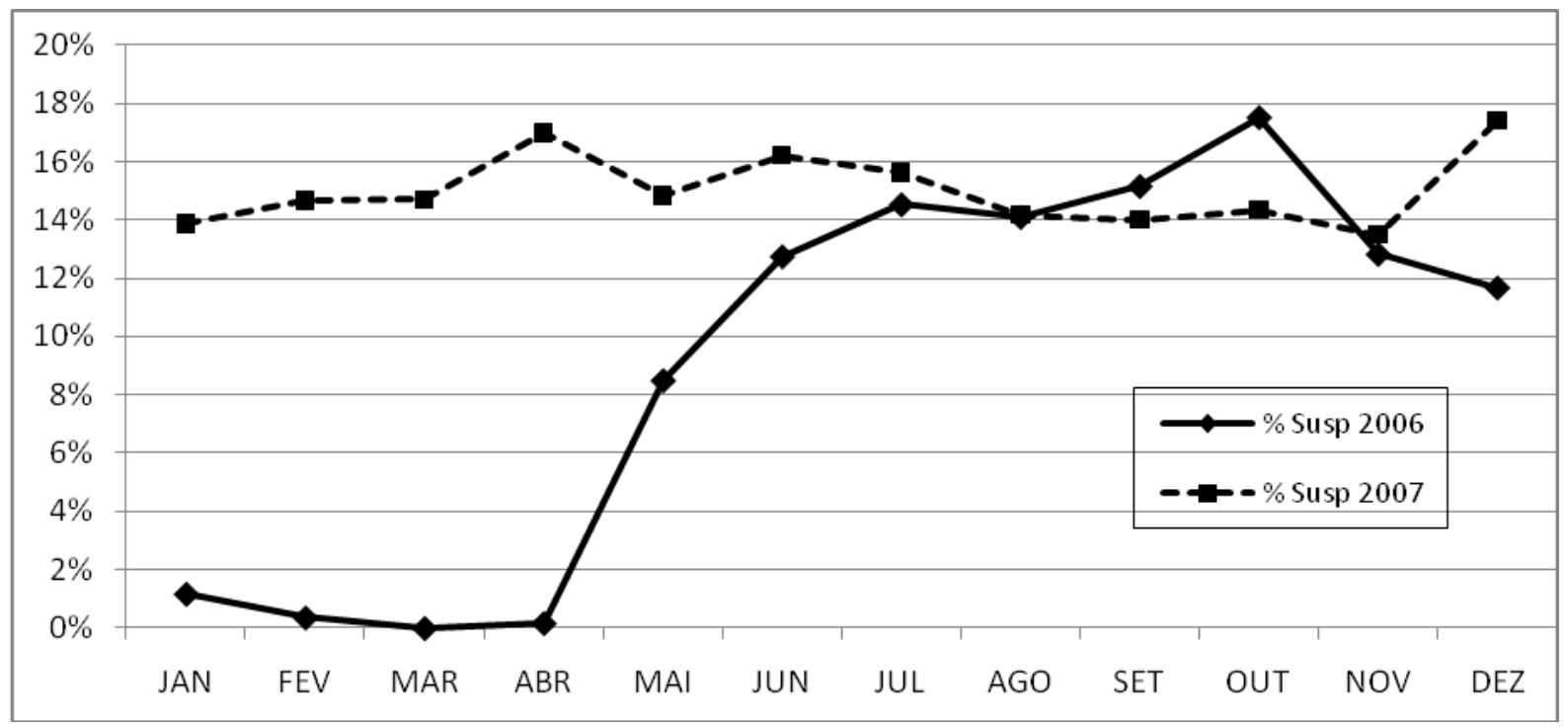

Gráfico 1 - Distribución de las suspensiones quirúrgicas según los meses del año. Uberaba (2006/2007).

En cuanto las características de los pacientes tuvieron cirugías canceladas en el periodo estudiado, la mayoría $1290(58,70 \%)$ era del género masculino y la media de edad fue de 46,12 años con variación de 2 meses a 97 años.

Entre las especialidades quirúrgicas, se verificó que la urología, la ortopedia, la CCP y el CAD presentaron mayores números de cancelación de sus procedimientos (Gráfico 2). 


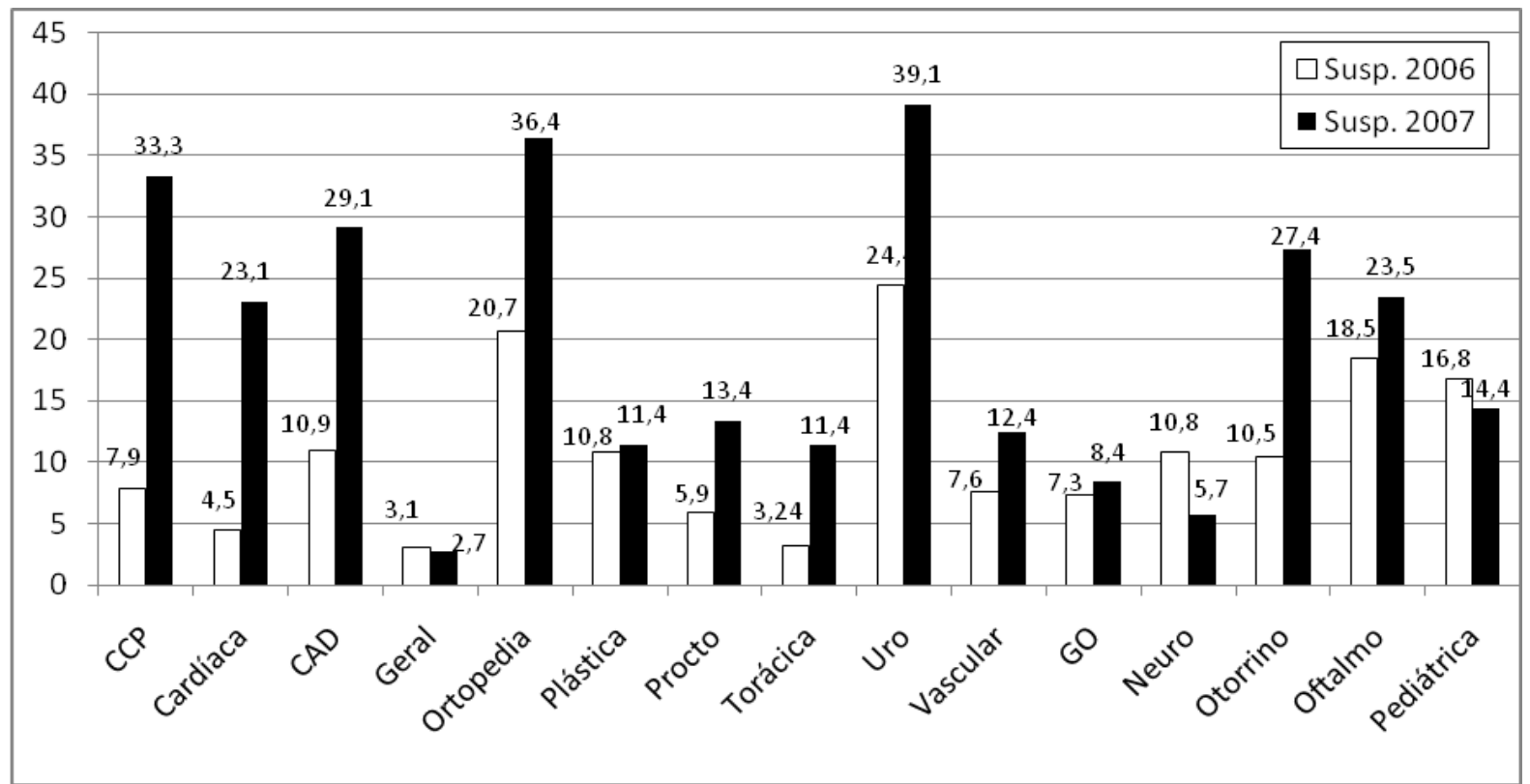

Gráfico 2 - Distribución de suspensión quirúrgica según las especialidades quirúrgicas. Uberaba (2006/2007)

Con relación a las justificativas identificadas para la cancelación de los procedimientos anestésico-quirúrgico, se observó que en la mayoría 1402 (63,78\%) de las cirugías suspendidas la justificación para la suspensión no fue registrada. El no ingreso del paciente, las condiciones clínicas inadecuadas y/o alteración de conducta médica y los problemas administrativos fueron las justificativas más frecuentes (Grafico 3).

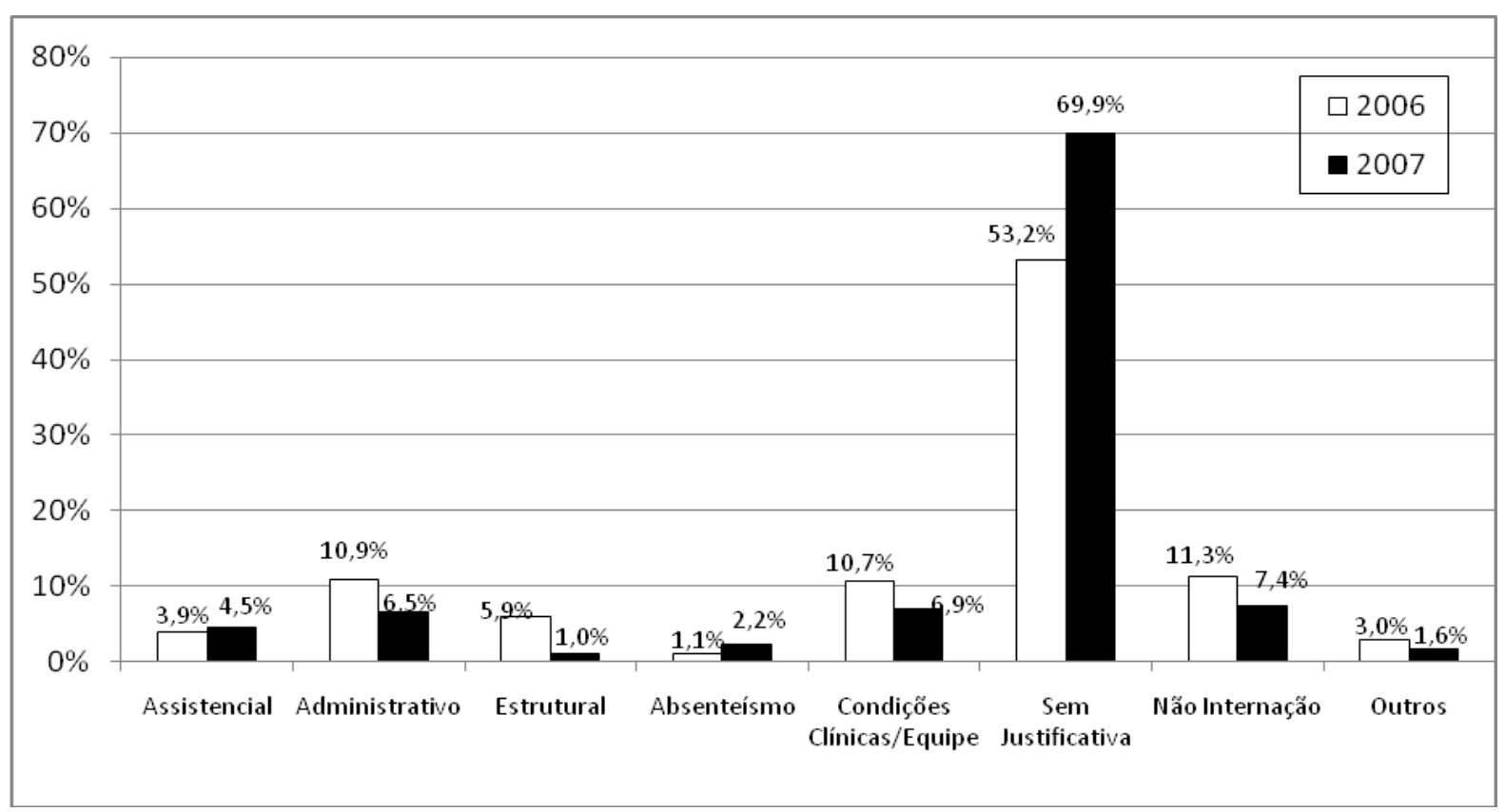

Gráfico 3 - Distribución de la tasa de suspensión quirúrgica según las justificativas (motivos) identificadas. Uberaba, (2006/2007)

En cuanto la cancelación de los procedimientos quirúrgicos por motivos asistenciales, se observaron 32 (34,04\%) por ausencia de evaluación pre-anestésica, 31 (32,98\%) por falta de 
ayunas, $19(20,21 \%)$ por análisis complementarias incompletos y $12(12,77 \%)$ por administración de medicaciones contraindicadas en el preoperatorio.

Se identificaron $179(8,14 \%)$ suspensiones quirúrgicas por motivos administrativos, siendo la deficiencia de los recursos materiales $(93 / 52,00 \%)$ y de recursos humanos $(25 / 14,00 \%)$ los motivos más frecuentes.

De los $61(2,77 \%)$ motivos relacionados con la estructura, la falta de cama fue la justificación principal para la cancelaciones quirúrgica $(49 / 80,30 \%)$.

En cuanto la ausencia del paciente, se observó que 40 (1,82\%) pacientes no comparecieron para el procedimiento quirúrgico programado, $194(8,82 \%)$ cirugías fueron suspendidas por no ingreso de los pacientes debido la guía y laudos de ingresos del SUS (falta de guía o de tarjeta del SUS o por cuota del SUS sobrepasada).

En cuanto los motivos relacionados a la condición clínica del paciente y/o alteración de la conducta médica, de las $182(8,30 \%)$ justificativas, la condición clínica inadecuada fue registrada en $158(86,80 \%)$ suspensiones, por alteración cardiovascular, respiratoria metabólica o presencia de cuadro infeccioso.

Se verificó que entre las $46(2,16 \%)$ suspensiones quirúrgicas por otros motivos, el retraso quirúrgico fue la principal $28(60,90 \%)$ justificación identificada.

\section{DISCUSIÓN}

En los últimos tiempos, la suspensión de cirugías ha sido considerada uno de los indicadores de calidad de la asistencia y ha sido el objetivo de estudio de algunos investigadores del área. Algunos estudios sugieren investigación sobre la ocurrencia de cancelación de cirugías realizadas por instituciones públicas y de enseñanza y cuestionaron si este evento es más común en este tipo de institución debido a sus características específicas ${ }^{(3)}$.

En este estudio se verificó que la tasa de suspensión quirúrgica fue de 14,14\%. La literatura revela mayores tasas variando entre $19,50 \%$ y $27,33 \%{ }^{(5)}$. Esta tasa podría ser reducida, a ejemplo de otros países. Una experiencia en el Reino Unido que utilizó la auditoría como proceso de intervención reveló una reducción de $42,90 \%$ de las cancelaciones quirúrgicas a través de la implantación de medidas asistenciales, administrativas y estructurales, como mejor distribución de las camas, previa evaluación clínica al citar y mejor comunicación con el paciente, discutiendo la fecha de la cirugía y su conveniencia además de orientación preoperatoria $^{(7)}$.

Seguramente, la implantación del SAEP también podría se adoptada, pues aparte de la evaluación preoperatoria del paciente posibilita la comunicación intra-sectorial y profesional para garantizar la continuidad del cuidado durante el ingreso, lo que viene siendo implementado por muchas instituciones brasileñas ${ }^{(8)}$. Cada institución debe establecer medidas específicas para reducir, cada vez más, la tasa de suspensión de cirugía en su servicio ${ }^{(5)}$.

Los principales motivos identificados en este estudio para la cancelación de los procedimientos quirúrgicos fueron administrativos, no ingreso del paciente, condiciones clínicas inadecuadas y/o alteraciones de conducta medica. Con todo, también es importante resaltar que en muchos casos no había el registro de estos motivos. Este hallazgo señala la 
necesidad de concienciación de los profesionales, pues sólo a partir del conocimiento de causa es como se consigue proponer soluciones.

Algunos autores ${ }^{(9,7)}$ destacan en sus estudios la ampliación de las discusiones sobre este aspecto por considerar que las cancelaciones de los procedimientos electivos son inevitables y minimizadas en cuanto estrategias para la mejora de la calidad adoptadas por las instituciones.

Entre estas, citan la necesidad de planificación y de control de la rutina quirúrgica a partir de los resultados relacionados con los motivos estructurales como la falta de camas, además de las condiciones clínicas inadecuadas de los pacientes y el absentismo, como las principales justificativas para la cancelación de los procedimientos quirúrgicos, lo que también fue evidenciado en esta investigación.

Otros estudios ${ }^{(12-13)}$ realizados tanto en Brasil comoo en otro países indican tasas elevadas de suspensiones quirúrgicas por condiciones clínicas inadecuadas de los pacientes y enfatizan la implementación de programas de orientación preoperatoria para obtener reducción de las tasas de suspensión de cirugías electivas. La carencia socioeconómica de los pacientes es considerada como uno de los factores para su ingreso aunque no esté en condiciones clínicas ideales para cirugía ${ }^{(5)}$.

El no ingreso del paciente después de llegar a la institución hospitalaria también fue otro motivo identificado para las cancelaciones quirúrgicas en esta investigación. En estos casos, los problemas descritos fueron la falta de tarjeta del SUS, la falta de guía de ingreso, la guía no autorizada, la guía incompleta, o laudo no aceptado y la cuota del SUS sobrepasada.

Para que los indicadores puedan ser utilizados como apoyo en el proceso de toma de decisión necesitan ser construidos de forma consistente. Esto demanda infraestructura de apoyo por medio de procesos sistematizados para obtención de datos confiables y generación de informaciones ${ }^{(3)}$.

Una de las exigencias para una asistencia de calidad y segura es que el sistema posea un canal de comunicación eficaz, permitiendo a los equipos transmitir y recibir informaciones de forma clara y correcta. Hay, sin embargo, falta de habilidades y conocimientos por parte de número importante de profesionales en lo que se refiere a la interacción con el paciente ${ }^{(14)}$.

Comunicación eficiente, evaluación y orientación al paciente electivo redujeron el desgaste para el paciente y para la propia institución ${ }^{(3)}$. La no asistencia del paciente a la cirugía programada presentó tasa inferior a los identificados en otros estudios ${ }^{(7,12)}$.

En cuanto las cuestiones administrativas, la falta de recursos materiales y humanos fue el principal motivo identificado, lo que diverge de los hallados en otros estudios ${ }^{(7)}$. Esto a lo mejor este relacionado con el modelo gerencial de recursos humanos y materiales adoptados por la institución. Algunos estudios ${ }^{(5,10)}$ apuntan fallos en la planificación de recursos tanto materiales como de personal.

Aunque no hayan sido evidenciados aspectos estructurales como unos de los principales motivos para la suspensión de las cirugías, la falta de camas fue la justificativa más identificada para justificar las cancelaciones de cirugías electivas. Esto también puede estar relacionado con fallos en la planificación. 


\section{CONCLUSIÓN}

Este estudio permitió evidenciar que la tasa de suspensión quirúrgica identificada fue $14,14 \%$. En lo que se refiere a las justificaciones para las suspensiones de las cirugías, las más frecuentes fueron relacionadas al no ingreso de los pacientes, condiciones clínicas insatisfactorias en el preoperatorio y/o alteraciones de la conducta médica y problemas administrativos de la institución hospitalaria.

Los hallazgos de esta investigación indican la necesidad de implementación de programas de concienciación de los profesionales para que puedan ser adoptadas medidas para garantizar la seguridad del paciente y la mejora de la calidad del servicio.

Resaltamos que lo incompleto de las informaciones en los historiales clínicos (no registro del motivo de cancelación de la cirugía) en algunos casos fue la limitación de este estudio. Esto sugiere la necesidad de implantación de programas específicos para los registros de estos datos, así como el de entrenamiento y concienciación para el relleno completo de los historiales clínicos. Sugerimos la realización de estudios prospectivos que aborden esta temática, así como investigaciones de intervenciones que evalúen medidas adoptadas para que estos déficits sean suprimidos en estos servicios.

\section{REFERENCIAS}

1. Cuocolo DF, Perroca MG. Reestruturação do quadro de pessoal de enfermagem e seu impacto sobre as horas de assistência. Rev Latino-Am Enfermagem [Internet]. 2010 mar/abr [acesso em 2011 Aug 25]; 18(2):31-39. Disponível em: http://www.scielo.br/pdf/rlae/v18n2/pt 06.pdf.

2. SiqueiraVT. Satisfação no trabalho: indicador de qualidade no gerenciamento de recursos humanos em enfermagem [dissertação]. São Paulo (SP): Escola de Enfermagem, Universidade de São Paulo. 2010.

3. Perroca MG, Jericó MC, Facudin SD. Monitorando o cancelamento de procedimentos cirúrgicos: indicador de desempenho organizacional. Rev. Esc. Enferm. USP. 2007a mar; 41( 1$): 113-19$.

4. Ministério da Saúde (Brasil), Secretaria Nacional de Organização e Desenvolvimento de Serviços de Saúde. Painel de Indicadores do Ministério da Saúde. Brasília: MS, 2006.

5. Landim FM, Paiva, FDS, Fiuza, MLT, Oliveira, EP, Pereira, JG, Siqueira et al. Análise dos fatores relacionados à suspensão de operações em um serviço de cirurgia geral de média complexidade. Rev. Col. Bras. Cir. [Internet]. 2009 jul/ago [acesso em 2011 Set 07]; 36(4):283-87. Disponível em: http://www.scielo.br/rcbc.

6. Bisinotto FMB, Pedrini Júnior M, Alves AAR, Andrade, MAPR Implantação do serviço de avaliação pré-anestésica em hospital universitário dificuldades e resultados. Rev. bras anestesiol. 2007 mar/abr; 57(2):167-76.

7. Perroca MG, Jericó MC, Facundin SD. Cancelamento cirúrgico em um hospital escola: implicações sobre o gerenciamento de custos. Rev. Latino-Am. Enfermagem. [Internet] 2007b set/out [acesso em 2011 Set 11]; 15(5):1018-24. Disponível em: http://www.scielo.br/pdf/rlae/v15n5/pt v15n5a20.pdf

8. Almeida RSS, Barros MM, Souza SEM. Implantação de um instrumento de coleta de dados perioperatórios. Rev. SOBECC. 2009 abr/jun; 14(2):41-46.

9. Zafar A, Mufti TS, Griffin S, Ahmed S, Ansari JA. Cancelled elective general surgical operations in Ayub teaching hospital. J Ayub Med Coll Abbottabad. [Internet] 2007 jul/sep [acesso em 2011 Set 07]; 19(3):64-7. Disponível em: www.http://nlm.nih.gov/pubmed/18444594. 
10. Garg R. Reasons for cancellation of cases on the day of surgery-a prospective study. Indian J Anaesth. [Internet] 2009 feb [acesso em 2011 Set 08]; 53(1): 35-9. Disponível em: http://www.ncbi.nlm.nih.gov/pmc/articles/PMC2900031/

11. Salles LF, Silva MJP. Correlação entre ansiedade e anéis de tensão. Rev Enf Referência. [Internet] 2011 mar [acesso em 2011 Set 20]; 3(3):7-13. Disponível em: http://www.esenfc.pt/rr/rr/index.php?id website $=3 \& d=1 \&$ target=DetalhesArtigo\&id artigo $=22$ 10\&id rev=9\&id edicao $=35$

12. Pitelkow E, Carvalho R. Cancelamento de cirurgias em um hospital da rede pública. Einstein. [Internet] 2008 [acesso em 2011 Set.06]; 6(4): 416-21. Disponível em: http://apps.einstein.br/revista/arquivos/PDF/988-Einsteinv6n4port416-421.pdf

13. Ahmed $\mathrm{T}$, Khan $\mathrm{M}$, Khan FA. Cancellation of surgery in patients attending the preoperative anaesthesia assessment clinic: a prospective study. J Pak Med Assoc. [Internet] 2009 Aug [ acesso em 2011 Sep 06]; 59(8):547-50. Disponível em: http://www.jpma.org.pk/full article text.php?article id=1776

14.Mourão CML, Albuquerque, MAS, Silva APS, Oliveira MS, Fernandes, AFC. Comunicação em enfermagem: uma revisão bibliográfica. Revista RENE. 2009 jul/set; 10(3):139-45 